\title{
Effect of Combining Ivabradine and $\beta$-Blockers: Focus on the Use of Carvedilol in the SHIFT Population
}

\author{
Edimar Alcides Bocchi ${ }^{a}$ Michael Böhm ${ }^{b}$ Jeffrey S. Borer ${ }^{c}$ lan Ford ${ }^{d}$ \\ Michel Komajda ${ }^{e}$ Karl Swedberg ${ }^{f}$ Luigi Tavazzi $^{9}$ on behalf of SHIFT \\ investigators
}

\begin{abstract}
${ }^{a}$ Heart Institute, Hospital das Clínicas da Faculdade de Medicina da University of São Paulo, São Paulo, Brazil; ${ }^{b}$ University of the Saarland, Homburg/Saar, Germany; ${ }^{C}$ University of New York Downstate Medical Center and College of Medicine, New York, N.Y., USA; ${ }^{d}$ Robertson Centre for Biostatistics, Glasgow, UK; ${ }^{\mathrm{C}} \mathrm{CHU}$ Pitié-Salpêtrière, Institut de Cardiologie, Paris, France; ${ }^{\mathrm{f}}$ Department of Molecular and Clinical Medicine, Institute of Medicine, Sahlgrenska University Hospital/Östra, University of Gothenburg, Gothenburg, Sweden; ${ }^{9}$ Fondazione IRCCS Policlinico San Mateo, Pavia, Italy
\end{abstract}

\section{Key Words}

$\beta$-Blockers · Chronic systolic heart failure $\cdot$ Heart rate

\begin{abstract}
Objectives: We explored the prescription of $\beta$-blockers with ivabradine in patients with systolic heart failure, focusing on the most frequently coprescribed $\beta$-blocker, carvedilol. Methods: We analyzed outcomes in SHIFT patients with systolic heart failure who were prescribed $\beta$-blockers (carvedilol, bisoprolol, metoprolol, or nebivolol) with ivabradine or placebo. Analysis was by intention to treat in patients prescribed a $\beta$-blocker at the time of the event. Results: Data were available for 2,596 patients receiving carvedilol, 1,483 bisoprolol, 1,424 metoprolol, and 197 nebivolol. Mean treatment duration was 19 months. There was no difference in the effect of ivabradine on the primary composite endpoint of cardiovascular death or heart failure hospitalization between the various $\beta$-blockers [hazard ratios (HR) for risk reduction, $0.75-0.89 ; p$ for interaction $=0.86$ ]. Patients prescribed carvedilol with ivabradine had lower rates of primary composite endpoint (HR 0.80,95\% Cl: 0.68-0.94), heart failure hospitalization (HR $0.73,95 \% \mathrm{Cl}: 0.61-0.88$ ), and cardio-
\end{abstract}

vascular hospitalization (HR $0.80,95 \% \mathrm{Cl}: 0.69-0.92)$ versus carvedilol with placebo. The dosage of carvedilol had no detectable effect and there were no unexpected safety issues. Conclusions: Whatever $\beta$-blocker was coprescribed with ivabradine, there were improvements in cardiovascular outcomes in patients with systolic heart failure, especially with the most prescribed $\beta$-blocker - carvedilol.

(C) 2015 S. Karger AG, Basel

\section{Introduction}

The management of heart failure has evolved tremendously in recent decades. Renin-angiotensin system (RAS) inhibitors and $\beta$-blockers are now widely used and have contributed to major therapeutic advances in heart failure. Despite this, the prognosis of patients with heart failure remains poor, with a median survival of 4.2 years without any discernible improvement over time [1]. There is clearly still room for improvement in management strategies and a relatively new agent, ivabradine, is a viable candidate for change in the management of systolic heart failure. Ivabradine acts via $I_{\mathrm{f}}$ inhibition in the

\section{KARGER 125}

(c) 2015 S. Karger AG, Base

0008-6312/15/1314-0218\$39.50/0

E-Mail karger@karger.com

www.karger.com/crd
Dr. Edimar Alcides Bocchi

Rua Oscar Freire 2077, Apto 161

São Paulo CEP 05409-011 (Brazil)

E-Mail eabocchi@yahoo.com 
sinoatrial node and reduces resting heart rate without altering other cardiac currents or hemodynamic parameters as well as without a negative inotropic effect $[2,3]$.

In 2010, the SHIFT (Systolic Heart Failure Treatment with the $I_{\mathrm{f}}$ Inhibitor Ivabradine Trial) investigators demonstrated that adding ivabradine to neurohormonal blockade improves mortality and morbidity in patients with systolic heart failure in sinus rhythm with a heart rate $\geq 70 \mathrm{bpm}$ [4]. Treatment with ivabradine was associated with a significant $18 \%$ reduction in the primary composite endpoint of cardiovascular death or hospitalization for worsening heart failure, a $26 \%$ reduction in hospitalization for worsening heart failure, and a $26 \%$ reduction in heart failure death. Since the SHIFT results were published, ivabradine has been granted the new indication and has become an increasingly important component in the management of chronic systolic heart failure [5], and has been included in international guidelines for the treatment of chronic heart failure, alongside other evidencebased therapies such as $\beta$-blockers and RAS inhibitors [5].

The large majority of the 6,505 patients in the SHIFT population were taking a background $\beta$-blocker at baseline (89\%) [4]. The SHIFT investigators have previously explored the effect of $\beta$-blocker dosage on outcomes [6]. They concluded that it was the heart rate reduction obtained with a $\beta$-blocker plus ivabradine - rather than the $\beta$-blocker dosage - that primarily determined the effect on outcomes. In the analysis described here, we set out to explore the effect of different individual $\beta$-blockers in combination with ivabradine on top of other evidence-based therapies in the SHIFT population, with a particular focus on the most frequently prescribed $\beta$-blocker - carvedilol [7].

\section{Methods}

\section{Study Design and Outcomes}

The design of the SHIFT trial has been described in great detail elsewhere $[4,8]$. Briefly, SHIFT was an event-driven randomized double-blind placebo-controlled trial carried out in 677 centers in 37 countries. Participants had moderate-to-severe heart failure (NYHA class II-IV) with left ventricular ejection fraction $\leq 35 \%$; they were in sinus rhythm and had a resting heart rate $\geq 70 \mathrm{bpm}$ (12-lead electrocardiography). Patients had to be on stable background treatment for $\geq 4$ weeks at entry, according to heart failure guidelines in force at the time the trial was performed [9]. Background treatment had been uptitrated as far as contraindications and tolerability would allow, and there was a particular emphasis on optimizing $\beta$-blocker dosage as close to the target as possible for each patient before initiation of study treatment. Against this background treatment, patients were randomly allocated to receive ivabradine (initiated at $5 \mathrm{mg}$ b.i.d., which could be adjusted up or down to 7.5 or $2.5 \mathrm{mg}$ b.i.d., according to resting heart rate and/or symptoms of bradycardia at 14 or 28 days, and then at every 4-month visit thereafter) or matching placebo.

In the post hoc analysis described here, we explored outcomes in SHIFT patients in subgroups divided according to the type of $\beta$-blocker they received at baseline (carvedilol, bisoprolol, metoprolol succinate or tartrate, or nebivolol). The outcome of interest was the primary endpoint, which is a composite of cardiovascular death and hospitalization for worsening heart failure. For the patients receiving the most frequently used $\beta$-blocker, carvedilol, we also investigated the following secondary endpoints: the individual components of the primary endpoint, heart failure death, cardiovascular hospitalization, and all-cause hospitalization, as well as safety data.

\section{Statistical Methods}

Baseline characteristics are presented according to treatment group ( $\beta$-blocker in combination with ivabradine or placebo) using means \pm SD for continuous variables and numbers of patients (\%) for categorical variables. Baseline characteristics were compared using a Kruskal-Wallis test (continuous variables) or a $\chi^{2}$ test (categorical variables).

The effect of the $\beta$-blocker in combination with ivabradine was analyzed in all randomized patients who received the combination of $\beta$-blocker with ivabradine or placebo at randomization. The analysis of events was performed exclusively while on the combination; in other words, only events that occurred between the first and last $(+2$ days $)$ concomitant intake of the $\beta$-blocker and the study treatment were taken into account. Hazard ratios (HRs) were estimated between treatment groups ( $\beta$-blocker in combination with ivabradine versus $\beta$-blocker in combination with placebo) based on an adjusted Cox's proportional hazards model with prognostic factors at baseline as covariates (NYHA class II/III or IV, left ventricular ejection fraction, ischemic etiology of heart failure, age, systolic blood pressure, heart rate, and creatinine clearance), and are presented with associated $95 \%$ CI. The associated $p$ values were given for the comparison between carvedilol in combination with ivabradine versus carvedilol in combination with placebo. Differences between $\beta$-blocker subgroups were tested using a likelihood ratio test for heterogeneity, comparing the model including the interaction term with the model not including the interaction term, to produce a $\mathrm{p}$ value for interaction. Treatment effect was also explored in patients receiving different dosages of carvedilol; the dose retained for each patient was that used for the longest time during the study. Due to the low size of these dosage subgroups, only the number of events and incidence were calculated.

Safety was assessed while on the combination in patients who had had at least one intake of carvedilol and randomized treatment during the study. Safety data were compared using two-sided Fisher's exact tests. Due to the exploratory nature of this analysis, no correction for multiplicity was applied. All statistical analyses were performed by the Biostatistics Division of the Institut de Recherches Internationales Servier using SAS ${ }^{\circledR}$ Software version 9.2.

\section{Results}

We analyzed 2,596 patients on carvedilol ( $45 \%$ of all patients receiving a $\beta$-blocker), 1,483 patients $(26 \%)$ on bisoprolol, 1,424 (25\%) on metoprolol (tartrate and/or 
Table 1. Baseline characteristics in 2,596 patients receiving carvedilol in combination with ivabradine or placebo at randomization

\begin{tabular}{|c|c|c|c|}
\hline & $\begin{array}{l}\text { Carvedilol + ivabradine } \\
(\mathrm{n}=1,318)\end{array}$ & $\begin{array}{l}\text { Carvedilol + placebo } \\
(\mathrm{n}=1,278)\end{array}$ & $\mathrm{p}$ \\
\hline \multicolumn{4}{|l|}{ Demographic characteristics } \\
\hline Age, years & $59.7 \pm 11.3$ & $59.2 \pm 11.8$ & 0.41 \\
\hline Male & $1,018(77)$ & $973(76)$ & 0.51 \\
\hline \multicolumn{4}{|l|}{ Ethnic origin } \\
\hline Caucasian & $1,171(89)$ & $1,141(89)$ & 0.83 \\
\hline Black & $25(2)$ & $26(2)$ & \\
\hline Asian & $73(6)$ & $61(5)$ & \\
\hline Other & $49(4)$ & $50(4)$ & \\
\hline Current smoker & $191(14)$ & $215(17)$ & 0.25 \\
\hline BMI, $\mathrm{kg} / \mathrm{m}^{2}$ & $28.1 \pm 5.2$ & $27.9 \pm 5.0$ & 0.41 \\
\hline \multicolumn{4}{|l|}{ Cardiac parameters } \\
\hline Heart rate, bpm & $79.5 \pm 9.2$ & $80.0 \pm 9.3$ & 0.15 \\
\hline Systolic blood pressure, $\mathrm{mm} \mathrm{Hg}$ & $119.9 \pm 16.0$ & $119.7 \pm 15.5$ & 0.70 \\
\hline Diastolic blood pressure, $\mathrm{mm} \mathrm{Hg}$ & $75.1 \pm 9.9$ & $75.0 \pm 9.7$ & 0.46 \\
\hline Left ventricular ejection fraction, $\%$ & $28.1 \pm 5.4$ & $28.2 \pm 5.5$ & 0.56 \\
\hline Creatinine clearance, $\mathrm{ml} / \mathrm{min} / 1.73 \mathrm{~m}^{2}$ & $74.8 \pm 23.0$ & $75.3 \pm 24.4$ & 0.80 \\
\hline \multicolumn{4}{|l|}{ NYHA class } \\
\hline Class II & $709(54)$ & $695(54)$ & 0.53 \\
\hline Class III & $598(45)$ & $567(44)$ & \\
\hline Class IV & $11(<1)$ & $16(1)$ & \\
\hline \multicolumn{4}{|l|}{ Medical history } \\
\hline Duration of heart failure, years & $3.6 \pm 4.3$ & $3.7 \pm 4.3$ & 0.50 \\
\hline \multicolumn{4}{|l|}{ Primary cause of heart failure } \\
\hline Ischemic & $800(61)$ & $761(60)$ & 0.55 \\
\hline Nonischemic & $518(39)$ & $517(40)$ & \\
\hline Myocardial infarction & $640(49)$ & $617(48)$ & 0.89 \\
\hline Hypertension & $819(62)$ & $798(62)$ & 0.87 \\
\hline Diabetes & $414(31)$ & $406(32)$ & 0.84 \\
\hline Stroke & $89(7)$ & $110(9)$ & 0.08 \\
\hline Atrial fibrillation and/or flutter & $93(7)$ & $96(8)$ & 0.66 \\
\hline \multicolumn{4}{|l|}{ Treatment at randomization } \\
\hline ACE inhibitor & $1,009(77)$ & $993(78)$ & 0.49 \\
\hline Angiotensin II receptor blocker & $213(16)$ & $184(14)$ & 0.21 \\
\hline Diuretics & $1,156(88)$ & $1,108(87)$ & 0.44 \\
\hline Mineralocorticoid receptor antagonist & $928(70)$ & $891(70)$ & 0.70 \\
\hline Digitalis & $345(26)$ & $336(26)$ & 0.95 \\
\hline At least one device & $56(4)$ & $65(5)$ & 0.31 \\
\hline Implanted ICD & $49(4)$ & $55(4)$ & 0.45 \\
\hline Implanted CRT & $16(1)$ & $22(2)$ & 0.28 \\
\hline
\end{tabular}

Data are number of patients $(\%)$ or means $\pm \mathrm{SD}$. $\mathrm{ACE}=$ Angiotensin-converting enzyme; $\mathrm{CRT}=$ cardiac resynchronization therapy; ICD = implantable cardioverter defibrillator.

succinate), and 197 (3\%) on nebivolol. There were 107 patients receiving other $\beta$-blockers who were not included in our analysis. The baseline characteristics of the largest subgroup, the 2,596 patients on the most prescribed $\beta$-blocker, carvedilol, are presented in table 1. No statisti- cal difference was shown between the patients allocated to ivabradine or placebo in any of the $\beta$-blocker subgroups (data not shown). Notably, the population was treated according to international guidelines. In the carvedilol subgroup, most patients received RAS inhibi- 
Table 2. Effect of $\beta$-blockers in combination with ivabradine vs. $\beta$-blockers in combination with placebo on the composite enpoint of cardiovascular death or hospitalization for worsening heart failure in patients receiving different $\beta$-blockers

\begin{tabular}{|c|c|c|c|c|c|c|}
\hline & \multicolumn{2}{|l|}{ Patients, $\mathrm{n}$} & \multicolumn{2}{|l|}{ Events, n (\%) } & $\mathrm{HR}(95 \% \mathrm{CI})$ & $\begin{array}{l}\mathrm{p} \text { for interaction } \\
\text { (heterogeneity) }\end{array}$ \\
\hline Carvedilol & 1,318 & 1,278 & $268(20)$ & $319(25)$ & $0.80(0.68-0.94)$ & 0.86 \\
\hline Bisoprolol & 720 & 763 & $112(16)$ & $137(18)$ & $0.89(0.69-1.15)$ & \\
\hline Metoprolol & 696 & 728 & $97(14)$ & $132(18)$ & $0.75(0.58-0.97)$ & \\
\hline
\end{tabular}

HR based on an adjusted Cox proportional hazards model with prognostic factors as covariates.

Table 3. Effect of treatment with carvedilol in combination with ivabradine $(n=1,318)$ vs. carvedilol in combination with placebo $(n=$ 1,278 ) on cardiovascular outcomes

\begin{tabular}{|c|c|c|c|c|}
\hline & \multicolumn{2}{|l|}{ Events, n (\%) } & \multirow[t]{2}{*}{$\operatorname{HR}(95 \% \mathrm{CI})$} & \multirow[t]{2}{*}{$\mathrm{p}$} \\
\hline & $\begin{array}{l}\text { Carvedilol + } \\
\text { ivabradine }\end{array}$ & $\begin{array}{l}\text { Carvedilol + } \\
\text { placebo }\end{array}$ & & \\
\hline \multicolumn{5}{|c|}{ Cardiovascular death or hospitalization for worsening } \\
\hline heart failure composite endpoint & $268(20)$ & $319(25)$ & $0.80(0.68-0.94)$ & 0.008 \\
\hline Cardiovascular death & $98(7)$ & $93(7)$ & $1.04(0.79-1.39)$ & 0.77 \\
\hline Heart failure hospitalization & $194(15)$ & $253(20)$ & $0.73(0.61-0.88)$ & 0.001 \\
\hline Cardiovascular hospitalization & $345(26)$ & $413(32)$ & $0.80(0.69-0.92)$ & 0.002 \\
\hline All-cause hospitalization & $445(34)$ & $481(38)$ & $0.89(0.78-1.02)$ & 0.084 \\
\hline Death from heart failure & $14(1.1)$ & $15(1.2)$ & - & - \\
\hline
\end{tabular}

HR based on an adjusted Cox proportional hazards model with prognostic factors as covariates.

tors $(77 \%$ on an angiotensin-converting enzyme inhibitor and $15 \%$ on an angiotensin II receptor blocker), diuretics (87\%), and mineralocorticoid antagonists (70\%). The mean duration of coprescription of carvedilol in combination with ivabradine or placebo was $19.2 \pm 9.0$ months.

After 28 days and after 1 year, heart rates were $63.8 \pm$ 10.7 and $64.3 \pm 11.1 \mathrm{bpm}$, respectively, for patients receiving carvedilol in combination with ivabradine versus $75.2 \pm 11.8$ and $74.4 \pm 12.6 \mathrm{bpm}$, respectively, for those receiving carvedilol in combination with placebo. Mean heart rate reduction at 28 days and at 1 year was $-15.7 \pm$ 10.5 and $-14.9 \pm 11.7 \mathrm{bpm}$ in patients receiving carvedilol in combination with ivabradine, respectively, compared with $-4.7 \pm 10.2$ and $-5.3 \pm 12.1 \mathrm{bpm}$ in patients receiving carvedilol in combination with placebo.

The favorable effect of $\beta$-blockers in combination with ivabradine compared with $\beta$-blockers in combination with placebo on the composite endpoint of cardiovascu- lar death or hospitalization for worsening heart failure was consistent whatever the $\beta$-blocker (HRs $0.75-0.89$, p for interaction $=0.86$; table 2 ). Patients prescribed carvedilol in combination with ivabradine had lower rates of the composite endpoint of cardiovascular death or hospitalization for worsening of heart failure (HR 0.80, 95\% CI: 0.68-0.94), heart failure hospitalization (HR 0.73, 95\% CI: 0.61-0.88), and cardiovascular hospitalization (HR 0.80, 95\% CI: 0.69-0.92) versus those prescribed carvedilol in combination with placebo (table 3 ). There was no difference in the rate of cardiovascular death (HR 1.04, 95\% CI: 0.79-1.39). There were 14 heart failure deaths $(1.1 \%)$ in the group on carvedilol in combination with ivabradine versus 15 (1.2\%) in the group on carvedilol in combination with placebo, which precluded any relevant conclusion with regard to this endpoint.

Of the 2,596 patients on carvedilol in combination with ivabradine or placebo, carvedilol dosages were evenly distributed throughout the dose range up to the recom- 
mended maximal target of $50 \mathrm{mg} /$ day [9]. A total of 417 (16\%) patients received $6.25 \mathrm{mg} /$ day, $626(24 \%)$ received $12.5 \mathrm{mg} /$ day, 587 (23\%) received $25 \mathrm{mg} /$ day, and 676 (26\%) received $50 \mathrm{mg} /$ day. The remaining 290 (11\%) patients received other dosages of carvedilol, which were generally between the lowest and highest of these four dosages. Carvedilol dosage was relatively stable during the study, and $88 \%$ of patients continued on the same dosage for the duration of coprescription ( $89 \%$ of the group on carvedilol in combination with ivabradine and $86 \%$ of the group on carvedilol in combination with placebo). In the $12 \%$ of patients who did change carvedilol dosage, most did so only once during the study. Whatever the dosage of carvedilol, ivabradine dosage was also relatively stable, with $25 \%$ patients receiving $5 \mathrm{mg}$ b.i.d. and $75 \%$ patients receiving $7.5 \mathrm{mg}$ b.i.d.

In all the carvedilol dosage subgroups, there were fewer cardiovascular deaths or hospitalization for worsening heart failure events with carvedilol in combination with ivabradine than carvedilol in combination with placebo [carvedilol $6.25 \mathrm{mg} /$ day, 67 (29.1\%) vs. 69 (36.9\%) events, respectively; carvedilol $12.5 \mathrm{mg} /$ day, $67(22.3 \%)$ vs. 85 (26.2\%) events; carvedilol $25 \mathrm{mg}$ /day, 63 (20.4\%) vs. 57 (20.5\%) events, and carvedilol $50 \mathrm{mg} /$ day, 48 (14.2\%) vs. $65(19.2 \%)$ events]. The low number of events in the individual dosage subgroups precluded any formal statistical analysis.

The safety of carvedilol in combination with ivabradine appeared to be in accordance with previous findings (table 4). There was no evidence for a relevant difference between the different dosages of carvedilol (data not shown).

\section{Discussion}

Our findings in the SHIFT population show that the coprescription of a $\beta$-blocker and ivabradine in patients with systolic heart failure is associated with an improvement in cardiovascular outcomes, regardless of the individual $\beta$-blocker coprescribed with ivabradine. This is in line with another analysis in this population [6], which reported that the effect on outcomes is determined primarily by the magnitude of heart rate reduction with $\beta$-blockers and ivabradine, as opposed to the actual dosage of the $\beta$-blocker. Our analysis takes this one step further by analyzing the effect of individual $\beta$-blockers, notably carvedilol. Carvedilol was the most frequently used $\beta$-blocker in patients with systolic heart failure in the SHIFT study. It was also the most widely prescribed
Table 4. Safety: selected emergent adverse events expected with carvedilol and ivabradine in patients who had taken at least one dose of the combination

\begin{tabular}{lccc}
\hline & Events, $\mathrm{n}(\%)$ & $\mathrm{p}$ \\
\cline { 2 - 3 } & $\begin{array}{l}\text { Carvedilol }+ \\
\text { ivabradine } \\
(\mathrm{n}=1,383)\end{array}$ & $\begin{array}{l}\text { Carvedilol }+ \\
\text { placebo } \\
(\mathrm{n}=1,361)\end{array}$ & \\
\cline { 1 - 2 } & & & \\
All emergent adverse & $1,036(75)$ & $984(72)$ & 0.13 \\
$\quad$ events & $331(24)$ & $388(29)$ & 0.007 \\
Heart failure & $118(9)$ & $85(6)$ & 0.024 \\
Atrial fibrillation & $67(3)$ & $8(<1)$ & $<0.001$ \\
Bradycardia & $33(2)$ & $6(<1)$ & $<0.001$ \\
Phosphenes & $30(1)$ & $41(3)$ & 0.12 \\
Hypotension & $14(1)$ & $7(<1)$ & 0.19 \\
Fatigue & $11(<1)$ & $4(<1)$ & 0.12 \\
Asthenia & $9(<1)$ & $17(1)$ & 0.12 \\
Hypertension & & & \\
Second- or third-degree & $7(<1)$ & $8(<1)$ & 0.80 \\
$\quad$ AV block & $5(<1)$ & $2(<1)$ & 0.45 \\
Blurred vision & $2(<1)$ & $3(<1)$ & 0.69 \\
Hyperglycemia & $1(<1)$ & 0 & 1.00 \\
QTc interval prolongation & $80(6)$ & $13(1)$ & $<0.001$ \\
Heart rate decrease & & & \\
\hline
\end{tabular}

$\mathrm{AV}=$ Atrioventricular.

$\beta$-blocker in a European registry including nearly 9,000 patients with systolic heart failure, in which $57 \%$ of patients were receiving carvedilol [7]. Similar rates of prescription of carvedilol were reported in the USA in a registry of about 6,000 patients with heart failure [10], as well as in the ESC-HF Pilot Survey, in which carvedilol was prescribed to $43 \%$ of patients in 12 European countries [11]. In our analysis, prescription of carvedilol in combination with ivabradine for a mean duration of 19 months was associated with an improvement in cardiovascular outcomes (a composite endpoint of cardiovascular death or hospitalization for worsening of heart failure, hospitalization for heart failure, cardiovascular hospitalization, and all-cause hospitalization) compared with carvedilol in combination with placebo.

Despite all the recent therapeutic advances, both mortality and morbidity in patients with heart failure remain unacceptably high [1]. Accordingly, there is a need to identify new therapeutic targets and associated strategies. Heart rate is now recognized as both a risk factor and a therapeutic target in heart failure, and its reduction has become an integral part of the management of risk factors in chronic systolic heart failure $[5,12]$. Reduction in resting heart rate is a common feature of the modes of action of both 
$\beta$-blockers and ivabradine, though $\beta$-blockers have other cardiovascular effects, such as a detrimental impact on myocardial contractility and hemodynamics, while ivabradine has no influence on other cardiac parameters [13, 14]. Furthermore, registry data suggest that heart rate is not adequately controlled in a large majority of patients, despite therapy with $\beta$-blockers $[15,16]$. In a large prospective observational study including 7,401 European patients with chronic heart failure, $56 \%$ had a resting heart rate $\geq 70$ bpm, despite the fact that $89 \%$ of patients were receiving a $\beta$-blocker [16]. This might be related to difficulties uptitrating $\beta$-blockers in many patients due to tolerability issues; in addition, some patients might have poor response due to $\beta$-receptor polymorphisms [17]. This implies that other strategies may be necessary to improve heart rate control in these patients. This may explain why ivabradine further reduced major risk associated with heart failure when added to evidence-based therapies including $\beta$-blockers. The SHIFT trial results presented herein show that the addition of ivabradine to a $\beta$-blocker is safe and effective in patients with chronic systolic heart failure. Moreover, the patients receiving ivabradine appeared no more or less likely to change dosage of carvedilol during the study, which suggests that use of ivabradine does not affect the use of carvedilol - or vice versa - and is reassuring regarding the safety and tolerability of this combination.

Our results have a number of implications for clinical practice. First, $\beta$-blockers are widely used in patients with heart failure, forming the cornerstone of their management together with RAS inhibitors. Our results also imply that $\beta$-blocker dosages in heart failure remain stable since $82 \%$ of patients on carvedilol stayed on the same dosage throughout the study. On the other hand, many patients have inadequate heart rate control despite the use of $\beta$-blockers. Insofar as ivabradine provides additional significant benefits when added to $\beta$-blockers, this should be considered in patients with chronic systolic heart failure in sinus rhythm and with a heart rate $\geq 70 \mathrm{bpm}$ in the presence of $\beta$-blockade as recommended by the latest heart failure guidelines [5]. Our analysis suggests that combination therapies may be applicable in heart failure, in particular a combination of carvedilol and ivabradine. One major advantage of combination therapies in chronic conditions is improved compliance [18], which implies better overall efficacy of treatment.

There are a number of limitations to our study. It suffers from all the limitations of a post hoc analysis, though the results are consistent with previous analyses of patients taking $\beta$-blockers in the SHIFT trial [6]. There was no randomized allocation of $\beta$-blockers, which were pre- scribed as a background treatment, using agents and dosages selected by the investigator. The relatively small size of the subgroups, notably for the various dosages of carvedilol, limited the power of the analysis and precluded any formal statistical analysis. In this exploratory analysis, no correction for multiplicity was applied.

\section{Conclusion}

Our findings suggest that the prescription of $\beta$-blocker in combination with ivabradine is associated with improvement in cardiovascular outcomes, regardless of the $\beta$-blocker used. In patients treated with carvedilol, the most frequently used $\beta$-blocker in the trial population, carvedilol in combination with ivabradine improved cardiovascular outcomes and was safe in patients with systolic heart failure in sinus rhythm with a resting heart rate $\geq 70 \mathrm{bpm}$.

\section{Conflict of Interest}

E.A.B. has received consulting fees from Baldacci and Servier; travel grants from Baldacci, Servier, Berlin Heart GmbH, and Novartis; fees for membership of steering committees from Servier and Novartis; fees for contracted research from Servier and Amgen, and honoraria from Servier. L.T. and I.F. received research grants and honoraria for conducting research as trial committee members sponsored by Servier. K.S. has received research support from Amgen and Pfizer. M.K. is a coprincipal investigator of the SHIFT trial and a member of the Speaker's Bureau for Servier. J.S.B. and K.S. are consultants to Servier and have received honoraria and fees from this company. K.S. is a consultant for Amgen, Novartis, Roche, and Vifor. M.B. has received consulting and speakers fees for Boehringer Ingelheim, Servier, and Medtronic.

References

1 McCullough PA, Philbin EF, Spertus JA, Kaatz S, Sandberg KR, Weaver WD: Confirmation of a heart failure epidemic: findings from the Resource Utilization among Congestive Heart Failure (REACH) study. J Am Coll Cardiol 2002;39:60-69.

2 DiFrancesco D: Funny channels in the control of cardiac rhythm and mode of action of selective blockers. Pharmacol Res 2006;53:399_ 406.

3 Deedwania P: Selective and specific inhibition of $I_{\mathrm{f}}$ with ivabradine for the treatment of coronary artery disease or heart failure. Drugs 2013;73:1569-1586

4 Swedberg K, Komajda M, Böhm M, et al: Ivabradine and outcomes in chronic heart failure (SHIFT): a randomised placebo-controlled trial. Lancet 2010;376:875-885. 
5 McMurray JJ, Adamopoulos S, Anker SD, et al: ESC guidelines for the diagnosis and treatment of acute and chronic heart failure 2012: The Task Force for the Diagnosis and Treatment of Acute and Chronic Heart Failure 2012 of the European Society of Cardiology. Developed in collaboration with the Heart Failure Association (HFA) of the ESC. Eur J Heart Fail 2012;14:803-869.

6 Swedberg K, Komajda M, Bohm M, et al: Effects on outcomes of heart rate reduction by ivabradine in patients with congestive heart failure: is there an influence of beta-blocker dose? J Am Coll Cardiol 2012;59:19381945.

7 Gjesing A, Schou M, Torp-Pedersen C, et al: Patient adherence to evidence-based pharmacotherapy in systolic heart failure and the transition of follow-up from specialized heart failure outpatient clinics to primary care. Eur J Heart Fail 2013;15:671-678.

8 Swedberg K, Komajda M, Böhm M, Borer JS, Ford I, Tavazzi L: Rationale and design of a randomized, double-blind, placebo-controlled outcome trial of ivabradine in chronic heart failure: the Systolic Heart Failure Treatment with the I(f) Inhibitor Ivabradine Trial (SHIFT). Eur J Heart Fail 2010;12:75-81.
9 Dickstein K, Cohen-Solal A, Filippatos G, et al: ESC Guidelines for the diagnosis and treatment of acute and chronic heart failure 2008. The Task Force for the Diagnosis and Treatment of Acute and Chronic Heart Failure 2008 of the European Society of Cardiology. Developed in collaboration with the Heart Failure Association of the ESC (HFA) and endorsed by the European Society of Intensive Care Medicine (ESICM). Eur J Heart Fail 2008;10:933-989.

10 Fonarow GC, Abraham WT, Albert NM, et al: Dosing of beta-blocker therapy before, during, and after hospitalization for heart failure (from Organized Program to Initiate Lifesaving Treatment in Hospitalized Patients with Heart Failure). Am J Cardiol 2008;102:15241529.

11 Maggioni AP, Dahlström U, Filippatos G, et al: EURObservational Research Programme: the Heart Failure Pilot Survey (ESC-HF Pilot). Eur J Heart Fail 2010;12:1076-1084.

12 Böhm M, Swedberg K, Komajda M, et al: Heart rate as a risk factor in chronic heart failure (SHIFT): the association between heart rate and outcomes in a randomised placebo-controlled trial. Lancet 2010;376: 886-894.
13 DiFrancesco D, Camm AJ: Heart rate lowering by specific and selective I(f) current inhibition with ivabradine. A new therapeutic perspective in cardiovascular disease. Drugs 2004;64:1757-1765.

14 Savelieva I, Camm AJ: I(f) inhibition with ivabradine: electrophysiological effects and safety. Drug Saf 2008;31:95-107.

15 Franke J, Wolter JS, Meme L, et al: Optimization of pharmacotherapy in chronic heart failure: is heart rate adequately addressed? Clin Res Cardiol 2013;102:23-31.

16 Maggioni AP, Anker SD, Dahlstrom U, et al: Are hospitalized or ambulatory patients with heart failure treated in accordance with European Society of Cardiology guidelines? Evidence from 12,440 patients of the ESC Heart Failure Long-Term Registry. Eur J Heart Fail 2013;15:1173-1184.

17 Terra SG, Pauly DF, Lee CR, et al: beta-Adrenergic receptor polymorphisms and responses during titration of metoprolol controlled release/extended release in heart failure. Clin Pharmacol Ther 2005;77:127-137.

18 Bangalore S, Kamalakkannan G, Parkar S, Messerli FH: Fixed-dose combinations improve medication compliance: a meta-analysis. Am J Med 2007;120:713-719. 